\title{
Formulation of value added beef meatball using tulsi (Ocimum sanctum) leaf extract as a source of natural antioxidant
}

\author{
Tahmina Siddiqua, Md. Anwar Hossain, Muckta Khan and Md. Abul Hashem
}

Department of Animal Science, Bangladesh Agricultural University, Mymensingh-2202, Bangladesh

\begin{tabular}{l}
\hline ARTICLE INFO \\
\hline Article history: \\
Received: 16 March 2018 \\
Accepted: 15 July 2018 \\
\hline Keywords: \\
Antioxidant, beef, meatball, \\
sensory, physicochemical, \\
microbial count, tulsi leaf extract \\
\hline Correspondence: \\
Md. Abul Hashem \\
(hashem_as@bau.edu.bd)
\end{tabular}

\begin{abstract}
The present study was undertaken to evaluate the effect of different levels of tulsi leaf extract on fresh and preserved beef meatballs. Four types of beef meatballs were formulated for this purpose. Meatballs were made with 0 (control), $0.1,0.2$ and $0.3 \%$ tulsi leaf extract, respectively and preserved at $-20^{\circ} \mathrm{C}$. Quality and safety evaluation of meatballs were determined by sensory, physicochemical, biochemical and microbiological tests. The analyses were conducted at $0,15^{\text {th }}, 30^{\text {th }}$ and $60^{\text {th }}$ days of interval. Considering $\mathrm{CP}$, tenderness, juiciness, overall acceptability, cooking loss, Free Fatty Acid (FFA), Per oxide Value (POV) and Thiobarbituric Acid Reactive Substances (TBARS) value, it can be concluded that tulsi leaf extract @ 0.1, 0.2 and $0.3 \%$ can be used in the formulation of beef meatball. In case of sensory evaluation $0.2 \%$ tulsi leaf extract is appreciated but on the basis of nutrient quality, physicochemical properties, biochemical analysis and microbial analysis $0.3 \%$ tulsi leaf extract is more satisfactory as a source of natural antioxidant than that of other treatment groups. Therefore, it may be concluded that $0.3 \%$ tulsi leaf extract can be added as a functional ingredients in beef meatball.
\end{abstract}

\section{Introduction}

Lipid peroxidation causes meat spoilage. It occurs during processing and storage when meatballs are exposed to oxygen, heat, and light (Fasseas et al., 2007). Antioxidant have an ability to prevent or reduce the oxidative damage of a tissue indirectly by enhancing natural defense of cell and/or directly by scavenging the free radical species (Verma et al., 2009). Over the years, synthetic antioxidants such as beta hydroxyl anisole, butyrate hydroxyl toluene and tertiary butyl hydroquinone etc. have been widely used to preserve meat and meat products (Fasseas et al., 2007). The use of these antioxidants is questionable since they have been discovered as toxic, mutagenic and carcinogenic effect to human and animal (Hayes et al., 2010). Hence there has been a growing interest in the use of natural antioxidants as alternative remedy for synthetic antioxidants. In addition, consumers have shifted their interest to natural antioxidants since they are considered safer than the synthetic antioxidants (Jung et al., 2010). It has also been reported that these natural antioxidants, especially of plant source, have greater application potential for consumer's acceptability, palatability, stability and shelf-life of meat products (Jung et al., 2010). Tulsi is grown in tropical and sub tropical including Indian Regions (Banerjee et al., 1996). This plant has been evaluated pharmacologically for antimicrobial, immunomodulatory, anti-stress, antiinflammatory, antipyretic, anti-asthmatic, hypoglycemic, hypotensive and analgesic activities. tulsi has been found to be utmost effective in various types of animal models (Chiang et al., 2005). Phenolics and flavonoids are the authentic antioxidants found in tulsi leaf that have been reported to be safe and bioactive (Sreelatha and Padma, 2009). Tulsi is a naturally occurring antioxidants that have many of the same functions as artificial antioxidants like butyrate hydroxyl anisole. It can increase shelf life of stored products without affecting qualities. It contains several compounds having multiple phenolic hydroxyl groups, such as apigenin, luteolin, vitexin, isovitexin, orientin, aesculetin, aesculin, chlorogenic acid and caffeic acid (Koushik and Gopal, 2013). The phenolic compounds, namely, cirsilineol, cirsimaritin, isothymusin, apigenin, rosmarinic acid and eugenol possess good antioxidant activity and have significant ability to scavenge highly reactive free radicals (Pandey and Madhuri, 2010). Tulsi, also known as Ocimum tenuiflorum, is revered as "Queen of herbs" due to its greater medicinal values and often consumed as herbal tea. Marked by its strong aroma and astringent taste, it is regarded as a kind of "elixir of life", as it is believed to promote longevity (Puri and Singh, 2002). Tulsi is known as "Queen of plant", "The mother medicine of nature" (Singh et al., 2010). No investigation on different levels of tulsi leaf extract on beef meatball has been carried out yet. With these view, the present study was undertaken to investigate the possibility of using different levels of tulsi leaf extract in beef meatballs to evaluate shelf-life, nutritional and microbiological status of beef meatball under different storage conditions.

\section{Materials and Methods}

\section{Collection of meat sample}

Boneless beef of $2.5 \mathrm{~kg}$ from freshly slaughtered cattle was collected from "Local market", Bangladesh Agricultural University, Mymensingh at 10.00 a.m. The 
meat sample was immediately transferred to the "Animal Science Laboratory" and stored at room temperature for 1 hour.

\section{Preparation of jar and other instruments}

All necessary instruments and jars or containers were cleaned with hot water and detergent powder and then autoclaved for sterilization and dried before starting the experimental activities.

\section{Sample preparation}

About $2.5 \mathrm{~kg}$ of fresh beef sample was taken for the preparation of beef meatball. First, the beef was cleaned with fresh water and the fat was trimmed with sharp knife. The beef sample was grinded properly and the spices, garam masala, salt, ice flakes, refined vegetable oil, refined wheat flower, sauce were mixed with grinded beef properly as per experimental design. There were four treatment groups as $0 \%\left(\mathrm{~T}_{0}\right), 0.1 \%\left(\mathrm{~T}_{1}\right), 0.2 \%$ $\left(\mathrm{T}_{2}\right)$, and $0.3 \%\left(\mathrm{~T}_{3}\right)$ tulsi leaf extract. The beef meatball of proper shape was prepared separately. It was then boiled in hot water for 2-3 minutes, water was removed from the meatball and fried in hot oil until reddish brown color was obtained. After frying the meatballs, they were packaged in polyethylene bags separately and kept into the freezer for further research at different day's of intervals.

\section{Sensory evaluation}

Different sensory attributes were examined. The meatball samples were evaluated by a trained 8-member panel. The sensory questionnaires measured intensity on a 5-point balanced semantic scale for the attributes of color, smell, tenderness, juiciness, and overall acceptability. Eight training sessions were held to familiarize the judges with the attributes to be evaluated and the scale to be used (Rubio et al., 2007). Prior to sample evaluation, all panelists participated in the orientation sessions to familiarize with the scale attributes (color, smell, juiciness, tenderness, and overall acceptability) of beef meatball using intensity scale. All samples were served in the Petri dishes. Sensory evaluation was accomplished at 0 day and repeated at 15, 30 and 60 days.

\section{Proximate composition}

Proximate composition such as Dry Matter (DM), Ether Extract (EE), Crude Protein (CP) and Ash were carried out according to the methods of AOAC (1995).

\section{Physicochemical properties measurement}

All $\mathrm{pH}$ value of raw, cooked and cooking loss meatball was measured using pH meter (Hanna HI99163) from raw meatball homogenate. It was prepared by blending 5 $\mathrm{g}$ of meat with $10 \mathrm{ml}$ distilled water.

\section{Biochemical analysis and microbial assessment}

Free Fatty Acid (FFA), Per Oxide Value (POV) and Thiobarbituric Acid Reactive Substances (TBARS) value were determined according to Sharma et al,
(2012). Total Viable Count (TVC), Total Coliform Count (TCC) and Total Yeast Mold Count (TYMC) were determined according to Ikhlas et al., (2011). All determination was done in triplicate and mean value was reported.

\section{Statistical analysis}

Data were analyzed using $4 \times 4$ factorial experiment in completely randomized design replicated three times per cell using SAS 9.1.3 version Statistical Discovery software, NC, USA. Duncan's Multiple Range Test (DMRT) test was used to determine the significance of differences among treatments means.

\section{Results and Discussion}

\section{Sensory evaluation}

It was found that sensory quality after fortification with tulsi leaf extracts was deteriorated with increased storage period. The range of overall observed color score at different treatment was 3.75 to 4.33 , flavor score was 3.75 to 4.17 , tenderness score was 3.58 to 4.17 , juiciness score was 3.83 to 4.00 and overall acceptability score was 3.83 to 4.08 (Table 1). The range of different day's intervals of overall observation of overall acceptability score was 3.08 to 4.58 . Among four treatments most preferable color and juiciness was observed from $0.2 \%$ $\left(\mathrm{T}_{2}\right)$, tenderness and overall acceptability was observed from $0.1 \%\left(\mathrm{~T}_{1}\right)$ and flavor was observed from $0.3 \%\left(\mathrm{~T}_{3}\right)$ tulsi leaf extract. Texture attributes viz, hardness, springiness, color, odor, gumminess and flavor as well as the nutritional quality of the product were significantly higher for clove and cardamom burfi. Sensory evaluation revealed that among different herbs, cardamon is highly preferred in burfi followed by ginger, turmeric , clove, curry leaves and tulsi (Prasad et al., 2017). It is in agreement with the present findings where tulsi leaf extract significantly affect different sensory attributes.

\section{Proximate components}

Overall DM content at different treatment was 53.99 to $59.29 \%$. The highest value was observed in 0 day and decreased gradually up to $60^{\text {th }}$ day (Table 2). The highest amount of DM content indicates this product is more preferable. DM content was increased with increased storage period because moisture loss was decreased with storage period. Similar results were reported for Indonesian traditional meatballs with a moisture content ranged from 69.52 to $71.17 \%$ (Purnomo and Rahardiyan 2008). The CP content at different treatments was 41.87 to 51.93. Synthetic antioxidant group contain more amount of CP than control group. The most preferable CP content was observed at $0.3 \%\left(\mathrm{~T}_{4}\right)$ tulsi leaf extracts group. The CP content at different days of interval was 23.32 to $22.24 \%$. The most preferable CP content was observed at 0 day and less preferable at $60^{\text {th }}$ day. The EE content at different treatments was 11.38 to $12.06 \%$. The most preferable EE content was observed at $0.2\left(\mathrm{~T}_{2}\right)$ 
and $0.3 \%\left(\mathrm{~T}_{3}\right)$ tulsi leaf extracts. The range of EE content at different days of interval was 11.82 to $11.89 \%$. The most preferable EE content was observed at 0 day and less preferable $\mathrm{EE}$ content was at $60^{\text {th }}$ day (Table 2). Serdaroglu et al. (2005) reported a similar fat content ranged from 7.9 to $8.8 \%$ in low-fat traditional Turkey koefte beef meatballs. Overall ash content at different treatments was 3.49 to $3.67 \%$. Control group contain higher amount of ash than treated with tulsi leaf extracts group except $T_{3}$.

\section{Physicochemical properties}

The range of overall observed raw $\mathrm{pH}$ at different treatments was 5.69 to $5.81 \%$. Among four treatments most preferable raw $\mathrm{pH}$ was observed at $0.3 \%\left(\mathrm{~T}_{3}\right)$ tulsi leaf extract (Table 3). The highest amount of raw $\mathrm{pH}$ indicated the higher preferability of the products for consumers' health. The raw $\mathrm{pH}$ among the treatments was decreased with increased storage period. The raw $\mathrm{p}^{\mathrm{H}}$ at different days of interval was 5.70 to $5.79 \%$. The most preferable raw $\mathrm{p}^{\mathrm{H}}$ was observed at $15^{\text {th }}$ day and less preferable was observed at $60^{\text {th }}$ day. Overall cooked $\mathrm{p}^{\mathrm{H}}$ at different treatments was 5.93 to $6.10 \%$. The most preferable cooked $\mathrm{p}^{\mathrm{H}}$ was observed at $0.3 \%\left(\mathrm{~T}_{3}\right)$ tulsi leaf extract. The most preferable cooked $\mathrm{p}^{\mathrm{H}}$ was observed at $30^{\text {th }}$ day and less preferable cooked $\mathrm{p}^{\mathrm{H}}$ at $60^{\text {th }}$ day. These results are similar to those of Sallam et al. (2004), who reported that storage time had a significant $(\mathrm{p}<0.05)$ effect on $\mathrm{pH}$ values, which tended to increase with storage time (up to 15 days with present finding). The overall cooking loss at different treatments was 23.97 to $24.95 \%$. The highest cooking loss was observed at $0.3 \%\left(\mathrm{~T}_{3}\right)$ tulsi leaf extract. The overall cooking loss at different days of interval was 22.35 to $26.32 \%$. The lowest cooking loss was observed at $60^{\text {th }}$ day and the highest cooking loss was observed at 0 day of observation. Cooking loss refers to the reduction of weight of meatballs during cooking process (Jama et al., 2008).

\section{Biochemical properties}

The biochemical effects of tulsi leaf extract on beef meatball is shown in Table 4. The overall FFA, POV and TBARS value at different treatment was 0.36 to $0.44 \%$., 4.47 to 4.20 and 0.49 to 0.59 , respectively. The overall FFA, POV and TBARS at different days of interval were 0.35 to $0.44 \%$., 4.03 to $4.59 \%$ and 0.40 to $0.69 \%$, respectively. The most preferable FFA value was observed at 0 day and less preferable FFA value was observed at $60^{\text {th }}$ day. The most preferable value was observed from $0.3 \%\left(\mathrm{~T}_{3}\right)$ tulsi leaf extract. The FFA value $(0.44)$ in the control group was significantly $(P<$ 0.01 ) higher than the values of the samples treated with $0.1,0.2$, and $0.3 \%$ tulsi leaf extracts. Lee and Kunz (2005) found that fermented sausages showed an increasing FFA content over time. It has been reported that these natural antioxidants, especially of plant source, have greater application potential for consumer's acceptability, palatability, stability and shelf-life of meat products (Jung et al., 2010). Throughout the storage time, POV were generally higher in control group compared to treatment groups (Table 4). The most preferable POV was observed at $0.3 \%\left(\mathrm{~T}_{3}\right)$ tulsi leaf extracts. The lowest amount of POV indicates that this product is most preferable for consumers health. The control sample showed a higher level of TBARS than samples treated with 0.1 , and $0.3 \%$ except $0.2 \%$ tulsi leaf extracts. This difference was especially significant $(p<0.01)$ after 60th days of storage time. Natural antioxidants, in particular polyphones, are the major plant compounds which have the ability to attenuate the oxidative damage of a tissue indirectly by enhancing natural defenses of cell and/or directly by scavenging the free radical species combat pathological disorders generated by physicochemical Reactive Oxygen Species (ROS) (Du et al., 2010). Antioxidants have an ability to prevent the oxidative damage of tissue indirectly by enhancing natural defenses of cell and directly by scavenging the free radical species (Verma et al., 2009). It has also been reported that these natural antioxidants, especially of plant source, have greater application potential for consumer's acceptability, palatability, stability and shelf-life of meat products (Jung et al., 2010). Tulsi leaf extracts can be a potential source of natural antioxidant which can be used in meat products.

\section{Microbiological assessment}

Effect of tulsi leaf extracts on beef meatball on microbial population is shown in Table 5. TVC value of fresh beef was 5.12 logs CFU/g beef, indicated good quality beef. The overall total viable count, TCC value and TYMC of beef meatball was 4.74 to $4.86,1.07$ to 1.13 , and 1.47 to 1.69 (log CFU/g), respectively at different treatment levels. The range of TVC value, TCC value and TYMC values at different days of interval was 4.50 to $5.13,1.01$ to 1.18 and1.11 to 1.89 , respectively. The TCC value of fresh beef was 1.13 logs CFU/g beef. Among these four treatments, the TCC in the control sample (1.13 logs $\mathrm{CFU} / \mathrm{g}$ ) was significantly higher than in the samples treated with $0.1,0.2$, and $0.3 \%$ of tulsi leaf extracts. The different superscript was observed from different treatment indicated that there were significant differences of TYMC values among these four treatment groups. Among four treatments, the total yeast-mold count in the control sample (1.69 log CFU/g) were significantly $(\mathrm{p}<0.01)$ higher than in the samples treated with, $0.1,0.2$, and $0.3 \%$ of tulsi leaf extracts. Some bacteria may be present in the product, but their growth is controlled under storage conditions (FernandezLopez et al., 2005). 
Siddiqua et al.

Table 1. Effect of tulsi leaf extract on sensory parameters in beef meatballs

\begin{tabular}{|c|c|c|c|c|c|c|c|c|c|}
\hline \multirow{2}{*}{ Parameters } & \multirow{2}{*}{ DI } & \multicolumn{4}{|c|}{ Treatments } & \multirow{2}{*}{ Mean } & \multicolumn{3}{|c|}{ Level of significance } \\
\hline & & $\mathrm{T}_{0}$ & $\mathrm{~T}_{1}$ & $\mathrm{~T}_{2}$ & $\mathrm{~T}_{3}$ & & Treat. & DI & $\mathrm{T} * \mathrm{DI}$ \\
\hline \multirow{7}{*}{ Color } & 0 & $4.67 \pm 0.33$ & $5.00 \pm 0.00$ & $4.67 \pm 0.33$ & $4.67 \pm 0.33$ & $4.75^{\mathrm{a}} \pm 0.25$ & \multirow{5}{*}{0.0906} & \multirow{5}{*}{$<0.0001$} & \multirow{5}{*}{0.2244} \\
\hline & 15 & $4.67 \pm 0.33$ & $4.00 \pm 0.00$ & $5.00 \pm 0.00$ & $4.00 \pm 0.58$ & $4.42^{\mathrm{a}} \pm 0.23$ & & & \\
\hline & 30 & $3.33 \pm 0.33$ & $3.67 \pm 0.33$ & $4.00 \pm 0.58$ & $3.33 \pm 0.33$ & $3.58^{\mathrm{b}} \pm 0.45$ & & & \\
\hline & 60 & $2.33 \pm 0.33$ & $3.67 \pm 0.33$ & $3.67 \pm 0.33$ & $3.33 \pm 0.33$ & $3.25^{\mathrm{b}} \pm 0.33$ & & & \\
\hline & Mean & $3.75^{\mathrm{a}} \pm 0.33$ & $4.08^{\mathrm{ab}} \pm 0.16$ & $4.33^{\mathrm{a}} \pm 0.31$ & $3.83^{\mathrm{ab}} \pm 0.39$ & & & & \\
\hline & 0 & $4.33 \pm 0.33$ & $4.33 \pm 0.33$ & $4.67 \pm 0.33$ & $4.67 \pm 0.33$ & $4.50^{\mathrm{a}} \pm 0.33$ & \multirow{5}{*}{0.3414} & \multirow{5}{*}{$<0.0001$} & \multirow{5}{*}{0.9622} \\
\hline & 15 & $4.33 \pm 0.33$ & $4.67 \pm 0.33$ & $4.33 \pm 0.33$ & $4.33 \pm 0.33$ & $4.42^{\mathrm{a}} \pm 0.33$ & & & \\
\hline \multirow{3}{*}{ Flavor } & 30 & 3.670 .33 & $4.00 \pm 0.58$ & $4.00 \pm 0.00$ & $4.33 \pm 0.33$ & $4.00^{\mathrm{a}} \pm 0.31$ & & & \\
\hline & 60 & $2.67 \pm 0.33$ & $3.33 \pm 0.33$ & $3.33 \pm 0.33$ & $3.33 \pm 0.33$ & $3.17^{\mathrm{b}} \pm 0.33$ & & & \\
\hline & Mean & $3.75^{\mathrm{a}} \pm 0.33$ & $4.08^{\mathrm{a}} \pm 0.39$ & $4.08^{\mathrm{a}} \pm 0.25$ & $4.17^{\mathrm{a}} \pm 0.33$ & & & & \\
\hline \multirow{6}{*}{ Tenderness } & 0 & $4.00 \pm 0.00$ & $4.67 \pm 0.33$ & $4.67 \pm 0.33$ & $4.33 \pm 0.33$ & $4.42^{\mathrm{a}} \pm 0.25$ & \multirow{5}{*}{0.0396} & \multirow{5}{*}{$<0.0001$} & \multirow{5}{*}{0.4427} \\
\hline & 15 & $4.00 \pm 0.00$ & $4.67 \pm 0.33$ & $4.67 \pm 0.33$ & $3.67 \pm 0.33$ & $4.25^{\mathrm{a}} \pm 0.25$ & & & \\
\hline & 30 & $3.67 \pm 0.33$ & $3.67 \pm 0.33$ & $3.67 \pm 0.33$ & $3.33 \pm 0.33$ & $3.58^{\mathrm{b}} \pm 0.33$ & & & \\
\hline & 60 & $2.67 \pm-0.33$ & $3.67 \pm 0.33$ & $3.33 \pm 0.33$ & $3.67 \pm 0.33$ & $3.33^{\mathrm{b}} \pm 0.33$ & & & \\
\hline & Mean & $3.58^{\mathrm{b}} \pm 0.16$ & $4.17^{\mathrm{a}} \pm 0.33$ & $4.08^{\mathrm{a}} \pm 0.33$ & $3.75^{\mathrm{ab}} \pm 0.33$ & & & & \\
\hline & 0 & $4.67 \pm 0.33$ & $4.33 \pm 0.33$ & $4.67 \pm 0.33$ & $4.33 \pm 0.33$ & $4.50^{\mathrm{a}} \pm 0.33$ & \multirow{5}{*}{0.8848} & \multirow{5}{*}{$<0.0001$} & \multirow{5}{*}{0.4226} \\
\hline \multirow{3}{*}{ Juiciness } & 15 & $5.00 \pm 0.00$ & $4.33 \pm 0.33$ & $4.67 \pm 0.33$ & $4.33 \pm 0.33$ & $4.58^{\mathrm{a}} \pm 0.25$ & & & \\
\hline & 30 & $3.33 \pm 0.33$ & $3.33 \pm 0.33$ & $3.67 \pm 0.33$ & $3.67 \pm 0.33$ & $5.50^{\mathrm{b}} \pm 0.33$ & & & \\
\hline & 60 & $2.33 \pm 0.33$ & $3.33 \pm 0.33$ & $3.00 \pm 0.33$ & $3.00 \pm 0.33$ & $3.00^{c} \pm 0.39$ & & & \\
\hline \multirow{6}{*}{$\begin{array}{c}\text { Overall } \\
\text { acceptability }\end{array}$} & Mean & $3.83^{\mathrm{a}} \pm 0.25$ & $3.83^{\mathrm{a}} \pm 0.33$ & $4.00^{\mathrm{a}} \pm 0.39$ & $3.92^{\mathrm{a}} \pm 0.33$ & & & & \\
\hline & 0 & $4.33 \pm 0.33$ & $4.67 \pm 0.33$ & $4.33 \pm 0.33$ & $4.67 \pm 0.33$ & $4.50^{\mathrm{a}} \pm 0.33$ & \multirow{5}{*}{0.6849} & \multirow{5}{*}{$<0.0001$} & \multirow{5}{*}{0.9493} \\
\hline & 15 & $4.33 \pm 0.33$ & $4.67 \pm 0.33$ & $4.67 \pm 0.33$ & $4.67 \pm 0.33$ & $4.58^{\mathrm{a}} \pm 0.33$ & & & \\
\hline & 30 & $3.67 \pm 0.33$ & $3.67 \pm 0.33$ & $3.67 \pm 0.33$ & $3.33 \pm 0.33$ & $3.58^{\mathrm{b}} \pm 0.33$ & & & \\
\hline & 60 & $3.00 \pm 0.58$ & $3.33 \pm 0.33$ & $3.33 \pm 0.33$ & $2.67 \pm 0.33$ & $3.08^{\mathrm{b}} \pm 0.39$ & & & \\
\hline & Mean & $3.83^{\mathrm{a}} \pm 0.39$ & $4.08^{\mathrm{a}} \pm 0.33$ & $4.00^{\mathrm{a}} \pm 0.33$ & $3.83^{\mathrm{a}} \pm 0.33$ & & & & \\
\hline
\end{tabular}

Sensory scores were 5 for excellent, 4 for very good, 3 for good, 2 for fair, and 1 for poor. Mean in each row having different superscript varies significantly at values $* \mathrm{P}<0.05$. Again, mean values having same superscript in each row did not differ significantly at $\mathrm{P}>0.05$. $\mathrm{T}_{0}=0 \%$ tulsi leaves extract, $\mathrm{T}_{1}=0.1 \%$ tulsi leaves extract, $\mathrm{T}_{2}=0.2 \%$ tulsi leaves extract, $\mathrm{T}_{3}=0.3 \%$ tulsi leaves extract, DI=Days of Intervals, Treat= Treatment, T*DI=Interaction of Treatment and Day Intervals.

Table 2. Effect of tulsi leaf extract on proximate components in beef meatballs

\begin{tabular}{|c|c|c|c|c|c|c|c|c|c|}
\hline \multirow{2}{*}{ Parameters } & \multirow{2}{*}{ DI } & \multicolumn{4}{|c|}{ Treatments } & \multirow{2}{*}{ Mean } & \multicolumn{3}{|c|}{ Level of significance } \\
\hline & & $\mathrm{T}_{0}$ & $\mathrm{~T}_{1}$ & $\mathrm{~T}_{2}$ & $\mathrm{~T}_{3}$ & & Treat. & DI & $\mathrm{T} * \mathrm{DI}$ \\
\hline \multirow{5}{*}{ DM\% } & 0 & $56.3 \pm 0.16$ & $55.49 \pm 0.15$ & $54.39 \pm 0.17$ & $52.44 \pm 0.13$ & $54.66^{\mathrm{d}} \pm 0.15$ & \multirow{5}{*}{$<0.0001$} & \multirow{5}{*}{$<0.0001$} & \multirow{5}{*}{$<0.0001$} \\
\hline & 15 & $58.87 \pm 0.05$ & $56.57 \pm 0.06$ & $54.67 \pm 0.07$ & $53.54 \pm 0.26$ & $55.91^{\mathrm{c}} \pm 0.11$ & & & \\
\hline & 30 & $59.48 \pm 0.19$ & $58.58 \pm 0.19$ & $55.41 \pm 0.10$ & $54.33 \pm 0.13$ & $56.95^{\mathrm{b}} \pm 0.15$ & & & \\
\hline & 60 & $61.53 \pm 0.16$ & $60.06 \pm 0.12$ & $57.66 \pm 0.07$ & $55.65 \pm 0.18$ & $58.73^{\mathrm{a}} \pm 0.13$ & & & \\
\hline & Mean & $59.29^{\mathrm{a}} \pm 0.14$ & $57.68^{\mathrm{b}} \pm 0.13$ & $55.53^{\mathrm{C}} \pm 0.10$ & $53.99^{\mathrm{d}} \pm 0.17$ & & & & \\
\hline \multirow{6}{*}{ СР\% } & 0 & $22.33 \pm 0.11$ & $23.09 \pm 0.07$ & $23.35 \pm 0.03$ & $23.53 \pm 0.06$ & $23.07^{\mathrm{a}} \pm 0.07$ & \multirow{5}{*}{$<0.0001$} & \multirow{5}{*}{$<0.0001$} & \multirow{5}{*}{$<0.0001$} \\
\hline & 15 & $22.19 \pm 0.03$ & $22.91 \pm 0.04$ & $23.16 \pm 0.02$ & $23.14 \pm 0.02$ & $22.85^{\mathrm{b}} \pm 0.03$ & & & \\
\hline & 30 & $22.04 \pm 0.05$ & $22.86 \pm 0.32$ & $22.92 \pm 0.03$ & $22.94 \pm 0.04$ & $22.69^{\mathrm{c}} \pm 0.11$ & & & \\
\hline & 60 & $21.69 \pm 0.04$ & $22.33 \pm 0.02$ & $22.27 \pm 0.01$ & $22.23 \pm 0.02$ & $22.13^{\mathrm{d}} \pm 0.07$ & & & \\
\hline & Mean & $22.06^{\mathrm{C}} \pm 0.06$ & $22.79^{\mathrm{b}} \pm 0.11$ & $22.92^{\mathrm{a}} \pm 0.02$ & $22.96^{\mathrm{a}} \pm 0.03$ & & & & \\
\hline & 0 & $12.20 \pm 0.08$ & $11.52 \pm 0.06$ & $11.84 \pm 0.03$ & $11.83 \pm 0.03$ & $11.85^{\mathrm{a}} \pm 0.05$ & \multirow{5}{*}{$<0.0001$} & \multirow{5}{*}{0.6444} & \multirow{5}{*}{$<0.0001$} \\
\hline \multirow{4}{*}{$\mathrm{EE} \%$} & 15 & $12.06 \pm 0.04$ & $11.49 \pm 0.02$ & $11.94 \pm 0.03$ & $11.91 \pm 0.03$ & $11.85^{\mathrm{a}} \pm 0.03$ & & & \\
\hline & 30 & $11.83 \pm 0.09$ & $11.31 \pm 0.05$ & $12.09 \pm 0.00$ & $12.04 \pm 0.04$ & $11.82^{\mathrm{a}} \pm 0.04$ & & & \\
\hline & 60 & $11.41 \pm 0.20$ & $11.20 \pm 0.02$ & $12.36 \pm 0.04$ & $12.22 \pm 0.03$ & $11.89^{\mathrm{a}} \pm 0.07$ & & & \\
\hline & Mean & $11.87^{\mathrm{b}} \pm 0.10$ & $11.38^{\mathrm{c}} \pm 0.04$ & $12.06^{\mathrm{a}} \pm 0.02$ & $12.06^{\mathrm{a}} \pm 0.03$ & & & & \\
\hline \multirow{5}{*}{ ASH\% } & 0 & $3.48 \pm 0.02$ & $3.47 \pm 0.04$ & $3.48 \pm 0.03$ & $3.53 \pm 0.01$ & $3.49^{\mathrm{b}} \pm 0.02$ & \multirow{5}{*}{0.0005} & \multirow{5}{*}{$<0.0001$} & \multirow{5}{*}{0.3534} \\
\hline & 15 & $3.64 \pm 0.04$ & $3.37 \pm 0.03$ & $3.56 \pm 0.02$ & $3.65 \pm 0.04$ & $3.55^{\mathrm{b}} \pm 0.03$ & & & \\
\hline & 30 & $3.51 \pm 0.11$ & $3.45 \pm 0.04$ & $3.51 \pm 0.01$ & $3.67 \pm 0.01$ & $3.54^{\mathrm{b}} \pm 0.04$ & & & \\
\hline & 60 & $3.78 \pm 0.09$ & $3.66 \pm 0.13$ & $3.65 \pm 0.03$ & $3.83 \pm 0.00$ & $3.73^{\mathrm{a}} \pm 0.06$ & & & \\
\hline & Mean & $3.60^{\mathrm{ab}} \pm 0.06$ & $3.49^{\mathrm{c}} \pm 0.06$ & $3.55^{\mathrm{bc}} \pm 0.22$ & $3.67^{\mathrm{a}} \pm 0.03$ & & & & \\
\hline
\end{tabular}

Mean in each row having different superscript varies significantly at values $\mathrm{P}<0.05 ., \mathrm{T}_{0}=0 \%$ tulsi leaf extract, $\mathrm{T}_{1}=0.1 \%$ tulsi leaves extract, $\mathrm{T}_{2}=0.2 \%$ tulsi leaves extract, $\mathrm{T}_{3}=0.3 \%$ tulsi leaf extract, DI=Day Intervals, Treat= Treatment, $\mathrm{T} * \mathrm{DI}=$ Interaction of Treatment and Day Intervals. 
Tulsi (Ocimum sanctum) leaves extract in beef meatball

Table 3. Effect of tulsi leaf extract on physicochemical parameters in beef meatballs

\begin{tabular}{|c|c|c|c|c|c|c|c|c|c|}
\hline \multirow{2}{*}{ Parameters } & \multirow{2}{*}{ DI } & \multicolumn{4}{|c|}{ Treatments } & \multirow{2}{*}{ Mean } & \multicolumn{3}{|c|}{ Level of significance } \\
\hline & & $\mathrm{T}_{0}$ & $\mathrm{~T}_{1}$ & $\mathrm{~T}_{2}$ & $\mathrm{~T}_{3}$ & & Treat. & $\mathrm{DI}$ & $\mathrm{T} * \mathrm{DI}$ \\
\hline \multirow{5}{*}{ Raw pH } & 0 & $5.68 \pm 0.01$ & $5.69 \pm 0.03$ & $5.72 \pm 0.01$ & $5.82 \pm 0.02$ & $5.73^{\mathrm{b}} \pm 0.02$ & \multirow{5}{*}{$<0.0001$} & \multirow{5}{*}{$<0.0001$} & \multirow{5}{*}{0.2146} \\
\hline & 15 & $5.72 \pm 0.02$ & $5.81 \pm 0.01$ & $5.80 \pm 0.02$ & $5.82 \pm 0.04$ & $5.79^{\mathrm{a}} \pm 0.02$ & & & \\
\hline & 30 & $5.68 \pm 0.00$ & $5.73 \pm 0.02$ & $5.72 \pm 0.02$ & $5.81 \pm 0.02$ & $5.74^{\mathrm{b}} \pm 0.01$ & & & \\
\hline & 60 & $5.67 \pm 0.03$ & $5.66 \pm 0.03$ & $5.67 \pm 0.01$ & $5.80 \pm 0.04$ & $5.70^{\mathrm{b}} \pm 0.03$ & & & \\
\hline & Mean & $5.69^{\mathrm{c}} \pm 0.01$ & $5.72^{\mathrm{b}} \pm 0.02$ & $5.73^{\mathrm{b}} \pm 0.01$ & $5.81^{\mathrm{a}} \pm 0.03$ & & & & \\
\hline \multirow{5}{*}{ Cooked pH } & 0 & $5.99 \pm 0.01$ & $5.99 \pm 0.01$ & $6.10 \pm 0.01$ & $6.24 \pm 0.03$ & $6.08^{\mathrm{b}} \pm 0.01$ & \multirow{5}{*}{$<0.0001$} & \multirow{5}{*}{$<0.0001$} & \multirow{5}{*}{0.0002} \\
\hline & 15 & $6.09 \pm 0.01$ & $6.19 \pm 0.02$ & $6.15 \pm 0.01$ & $6.14 \pm 0.00$ & $6.12^{\mathrm{a}} \pm 0.01$ & & & \\
\hline & 30 & $5.93 \pm 0.00$ & $5.96 \pm 0.01$ & $6.07 \pm 0.04$ & $6.06 \pm 0.04$ & $6.00^{\mathrm{C}} \pm 0.02$ & & & \\
\hline & 60 & $5.73 \pm 0.02$ & $5.82 \pm 0.01$ & $5.91 \pm 0.01$ & $5.93 \pm 0.04$ & $5.85^{\mathrm{d}} \pm 0.02$ & & & \\
\hline & Mean & $5.93^{\mathrm{d}} \pm 0.01$ & $5.97^{\mathrm{C}} \pm 0.01$ & $6.06^{\mathrm{b}} \pm 0.02$ & $6.10^{\mathrm{a}} \pm 0.03$ & & & & \\
\hline \multirow{5}{*}{$\begin{array}{l}\text { Cooking } \\
\text { loss (\%) }\end{array}$} & 0 & $27.21 \pm 0.17$ & $26.12 \pm 0.06$ & $26.01 \pm 0.01$ & $25.94 \pm 0.02$ & $26.32^{\mathrm{a}} \pm 0.06$ & \multirow{5}{*}{0.0071} & \multirow{5}{*}{$<0.0001$} & \multirow{5}{*}{0.0589} \\
\hline & 15 & $25.71 \pm 0.23$ & $24.90 \pm 0.07$ & $25.93 \pm 0.01$ & $25.87 \pm 0.01$ & $25.60^{\mathrm{b}} \pm 0.08$ & & & \\
\hline & 30 & $23.80 \pm 0.46$ & $23.94 \pm 0.29$ & $23.27 \pm 0.72$ & $22.11 \pm 0.07$ & $23.28^{c} \pm 0.38$ & & & \\
\hline & 60 & $23.08 \pm 0.75$ & $22.39 \pm 0.78$ & $21.96 \pm 0.18$ & $21.96 \pm 0.38$ & $22.35^{\mathrm{d}} \pm 0.52$ & & & \\
\hline & Mean & $24.95^{\mathrm{a}} \pm 0.40$ & $24.34^{\mathrm{b}} \pm 0.30$ & $24.29^{\mathrm{b}} \pm 0.23$ & $23.97^{\mathrm{b}} \pm 0.12$ & & & & \\
\hline
\end{tabular}

Mean in each row having different superscript varies significantly at values $\mathrm{p}<0.05$. $\mathrm{T}_{0}=0 \%$ tulsi leaves extract, $\mathrm{T}_{1}=0.1 \%$ tulsi leaf extract, $\mathrm{T}_{2}=0.2 \%$ tulsi leaf extract, $\mathrm{T}_{3}=0.3 \%$ tulsi leaf extract, DI=Day Intervals, Treat $=$ Treatment, $\mathrm{T}^{*} \mathrm{DI}=$ Interaction of Treatment and Day Intervals.

Table 4. Effect of tulsi leaf extract on biochemical parameters in beef meatballs

\begin{tabular}{|c|c|c|c|c|c|c|c|c|c|}
\hline \multirow{2}{*}{ Parameters } & \multirow{2}{*}{ DI } & \multicolumn{4}{|c|}{ Treatments } & \multirow{2}{*}{ Mean } & \multicolumn{3}{|c|}{ Level of significance } \\
\hline & & $\mathrm{T}_{0}$ & $\mathrm{~T}_{1}$ & $\mathrm{~T}_{2}$ & $\mathrm{~T}_{3}$ & & Treat. & DI & $\mathrm{T} * \mathrm{DI}$ \\
\hline \multirow{4}{*}{$\begin{array}{c}\text { FFA } \\
(\%)\end{array}$} & 0 & $0.38 \pm 0.00$ & $0.34 \pm 0.00$ & $0.34 \pm 0.00$ & $0.33 \pm 0.00$ & $0.35^{\mathrm{d}} \pm 0.00$ & \multirow{5}{*}{$<0.0001$} & \multirow{5}{*}{$<0.0001$} & \multirow{5}{*}{$<0.0001$} \\
\hline & 15 & $0.40 \pm 0.01$ & $0.38 \pm 0.00$ & $0.34 \pm 0.00$ & $0.33 \pm 0.00$ & $0.37^{\mathrm{C}} \pm 0.002$ & & & \\
\hline & 30 & $0.46 \pm 0.01$ & $0.41 \pm 0.00$ & $0.36 \pm 0.00$ & $0.35 \pm 0.00$ & $0.39^{\mathrm{b}} \pm 0.002$ & & & \\
\hline & 60 & $0.52 \pm 0.01$ & $0.43 \pm 0.00$ & $0.42 \pm 0.00$ & $0.41 \pm 0.00$ & $0.44^{\mathrm{a}} \pm 0.002$ & & & \\
\hline \multirow{6}{*}{$\begin{array}{c}\text { POV } \\
(\mathrm{meq} / \mathrm{kg})\end{array}$} & Mean & $0.44^{\mathrm{a}} \pm 0.01$ & $0.39^{\mathrm{b}} \pm 0.00$ & $0.37^{\mathrm{C}} \pm 0.00$ & $0.36^{\mathrm{d}} \pm 0.00$ & & & & \\
\hline & 0 & $4.06 \pm 0.06$ & $3.97 \pm 0.01$ & $4.03 \pm 0.01$ & $4.03 \pm 0.01$ & $4.03^{\mathrm{d}} \pm 0.02$ & \multirow{5}{*}{$<0.0001$} & \multirow{5}{*}{$<0.0001$} & \multirow{5}{*}{$<0.0001$} \\
\hline & 15 & $4.28 \pm 0.06$ & $4.13 \pm 0.02$ & $4.24 \pm 0.02$ & $4.19 \pm 0.02$ & $4.19^{\mathrm{c}} \pm 0.03$ & & & \\
\hline & 30 & $4.71 \pm 0.01$ & $4.51 \pm 0.01$ & $4.41 \pm 0.02$ & $4.19 \pm 0.04$ & $4.45^{\mathrm{b}} \pm 0.02$ & & & \\
\hline & 60 & $4.85 \pm 0.01$ & $4.56 \pm 0.04$ & $4.47 \pm 0.03$ & $4.48 \pm 0.09$ & $4.59^{\mathrm{a}} \pm 0.04$ & & & \\
\hline & Mean & $4.47^{\mathrm{a}} \pm 0.03$ & $4.29^{\mathrm{b}} \pm 0.02$ & $4.29^{\mathrm{b}} \pm 0.02$ & $4.20^{\mathrm{c}} \pm 0.04$ & & & & \\
\hline \multirow{5}{*}{$\begin{array}{c}\text { TBARS } \\
\text { (mg-MA/kg }\end{array}$} & 0 & $0.41 \pm 0.00$ & $0.40 \pm 0.00$ & $0.49 \pm 0.00$ & $0.49 \pm 0.00$ & $0.40^{\mathrm{d}} \pm 0.00$ & \multirow{5}{*}{$<0.0001$} & \multirow{5}{*}{$<0.0001$} & \multirow{5}{*}{$<0.0001$} \\
\hline & 15 & $0.48 \pm 0.00$ & $0.43 \pm 0.00$ & $0.42 \pm 0.00$ & $0.42 \pm 0.00$ & $0.44^{\mathrm{C}} \pm 0.00$ & & & \\
\hline & 30 & $0.61 \pm 0.00$ & $0.58 \pm 0.02$ & $0.54 \pm 0.00$ & $0.51 \pm 0.01$ & $0.56^{\mathrm{b}} \pm 0.01$ & & & \\
\hline & 60 & $0.80 \pm 0.00$ & $0.70 \pm 0.01$ & $0.63 \pm 0.00$ & $0.62 \pm 0.00$ & $0.69^{\mathrm{a}} \pm 0.00$ & & & \\
\hline & Mean & $0.58^{\mathrm{a}} \pm 0.00$ & $0.53^{\mathrm{b}} \pm 0.01$ & $0.59^{\mathrm{c}} \pm 0.00$ & $0.49^{\mathrm{d}} \pm 0.00$ & & & & \\
\hline
\end{tabular}

Mean in each row having different superscript varies significantly at values $\mathrm{P}<0.05$. $\mathrm{T}_{0}=0 \%$ tulsi leaves extract, $\mathrm{T}_{1}=0.1 \%$ tulsi leaf extract, $\mathrm{T}_{2}=0.2 \%$ tulsi leaf extract, $\mathrm{T}_{3}=0.3 \%$ tulsi leaf extract, DI $=$ Day Intervals, Treat $=$ Treatment, $\mathrm{T} * \mathrm{DI}=$ Interaction of Treatment and Day Intervals FFA = Free Fatty Acid, POV = Per Oxide Value, TBARS = Thiobarbituric Acid Reactive Substances.

Table 5. Effect of tulsi leaf extract on microbial population in beef meatballs

\begin{tabular}{|c|c|c|c|c|c|c|c|c|c|}
\hline \multirow{2}{*}{ Parameters } & \multirow{2}{*}{ DI } & \multicolumn{4}{|c|}{ Treatments } & \multirow{2}{*}{ Mean } & \multicolumn{3}{|c|}{ Level of significance } \\
\hline & & $\mathrm{T}_{0}$ & $\mathrm{~T}_{1}$ & $\mathrm{~T}_{2}$ & $\mathrm{~T}_{3}$ & & Treat. & DI & $\mathrm{T} * \mathrm{DI}$ \\
\hline \multirow{5}{*}{$\begin{array}{c}\text { TVC } \\
(\log \mathrm{CFU} / \mathrm{g})\end{array}$} & 0 & $4.65 \pm 0.03$ & $4.57 \pm 0.04$ & $4.43 \pm 0.00$ & $4.37 \pm 0.02$ & $4.50^{\mathrm{C}} \pm 0.02$ & \multirow{5}{*}{0.2396} & \multirow{5}{*}{$<0.0001$} & \multirow{5}{*}{0.5151} \\
\hline & 15 & $4.87 \pm 0.01$ & $4.68 \pm 0.01$ & $4.71 \pm 0.02$ & $4.72 \pm 0.03$ & $4.74^{\mathrm{b}} \pm 0.02$ & & & \\
\hline & 30 & $4.86 \pm 0.08$ & $4.78 \pm 0.01$ & $4.76 \pm 0.01$ & $4.72 \pm 0.00$ & $4.78^{\mathrm{b}} \pm 0.02$ & & & \\
\hline & 60 & $5.06 \pm 0.31$ & $4.43 \pm 0.01$ & $5.19 \pm 0.00$ & $5.14 \pm 0.00$ & $5.13^{\mathrm{a}} \pm 0.08$ & & & \\
\hline & Mean & $4.86^{\mathrm{a}} \pm 0.17$ & $4.79^{\mathrm{a}} \pm 0.02$ & $4.77^{\mathrm{a}} \pm 0.01$ & $4.74^{\mathrm{a}} \pm 0.01$ & & & & \\
\hline \multirow{5}{*}{$\begin{array}{c}\text { TCC } \\
(\log \mathrm{CFU} / \mathrm{g})\end{array}$} & 0 & $1.19 \pm 0.01$ & $1.17 \pm 0.01$ & $1.20 \pm 0.01$ & $1.16 \pm 0.01$ & $1.18^{\mathrm{a}} \pm 0.01$ & \multirow{5}{*}{0.0026} & \multirow{5}{*}{$<0.0001$} & \multirow{5}{*}{0.0795} \\
\hline & 15 & $1.17 \pm 0.03$ & $1.12 \pm 0.00$ & $1.15 \pm 0.00$ & $1.12 \pm 0.00$ & $1.14^{\mathrm{b}} \pm 0.01$ & & & \\
\hline & 30 & $1.11 \pm 0.00$ & $1.19 \pm 0.02$ & $1.05 \pm 0.01$ & $1.06 \pm 0.03$ & $1.08^{\mathrm{C}} \pm 0.26$ & & & \\
\hline & 60 & $1.04 \pm 0.01$ & $1.06 \pm 0.03$ & $0.98 \pm 0.03$ & $0.96 \pm 0.03$ & $1.01^{\mathrm{d}} \pm 0.02$ & & & \\
\hline & Mean & $1.13^{\mathrm{a}} \pm 0.01$ & $1.11^{\mathrm{ab}} \pm 0.01$ & $1.09^{\mathrm{bc}} \pm 0.01$ & $1.07^{\mathrm{C}} \pm 0.02$ & & & & \\
\hline \multirow{5}{*}{$\begin{array}{c}\text { TYMC } \\
(\log \text { CFU/g) }\end{array}$} & 0 & $1.96 \pm 0.02$ & $1.87 \pm 0.02$ & $1.86 \pm 0.00$ & $1.87 \pm 0.00$ & $1.89^{\mathrm{a}} \pm 0.01$ & \multirow{5}{*}{$<0.0001$} & \multirow{5}{*}{$<0.0001$} & \multirow{5}{*}{$<0.0001$} \\
\hline & 15 & $1.76 \pm 0.01$ & $1.56 \pm 0.01$ & $1.62 \pm 0.00$ & $1.56 \pm 0.02$ & $1.63^{\mathrm{b}} \pm 0.01$ & & & \\
\hline & 30 & $1.55 \pm 0.02$ & $1.38 \pm 0.02$ & $1.42 \pm 0.02$ & $1.39 \pm 0.02$ & $1.43^{\mathrm{C}} \pm 0.02$ & & & \\
\hline & 60 & $1.12 \pm 0.02$ & $1.08 \pm 0.01$ & $1.12 \pm 0.00$ & $1.11 \pm 0.01$ & $1.11^{\mathrm{d}} \pm 0.01$ & & & \\
\hline & Mean & $1.69^{\mathrm{a}} \pm 0.02$ & $1.47^{\mathrm{C}} \pm 0.01$ & $1.50^{\mathrm{b}} \pm 0.00$ & $1.48^{\mathrm{C}} \pm 0.01$ & & & & \\
\hline
\end{tabular}

Mean in each row having different superscript varies significantly at values $\mathrm{P}<0.05$. $\mathrm{T}_{0}=0 \%$ tulsi leaves extract, $\mathrm{T}_{1}=0.1 \%$ tulsi leaves extract, $\mathrm{T}_{2}=0.2 \%$ tulsi leaves extract, $\mathrm{T} 3=0.3 \%$ tulsi leaves extract, $\mathrm{DI}=$ Day Intervals, Treat $=$ Treatment, $\mathrm{T} * \mathrm{DI}=\mathrm{Interaction}$ of Treatment and Day Intervals, TVC = Total Viable Count, TCC $=$ Total Coliform Count, TYMC = Total Yeast-Mold Count. 


\section{Conclusion}

From the study it may be concluded that $0.3 \%$ of tulsi leaf extracts as natural antioxidant may be used in beef meatballs preparation. On the basis of sensory evaluation, physicochemical properties, biochemical analysis and microbial assessment indicated that $0.3 \%$ tulsi leaf extracts showed better results in the preparation of beef meatball compare to control and other two treatments.

\section{Acknowledgement}

We would like to express our appreciation to the Bangladesh Agricultural University Research System (BAURES), Mymensingh and Ministry of Science and Technology (MOST), Bangladesh for funding this research and their continuous encouragement to complete the study successfully.

\section{References}

AOAC, 1995. Official method of analysis. $17^{\text {th }}$ edition. Association of Official Analytical Chemists, Washington, D.C. USA.

Du, J., Zeng Y., Wang H. Qian, Y., Li, H., Chen, Q., Chen, W. and Cui, J. 2010. CuZnSOD gene expression and its relationship with anti-oxidative capacity and pork quality. South African Journal of Animal Science. 40: 265-272.

Fasseas, M. K., Mountzouris, C. K., Tarantilis, P. A., Polissiou, M. and Zervas, G. 2007. Antioxidant activity in meat treated with oregano and sage essential oils. Food Chemistry.106: 1188-1194.

Fernández-López, J., Zhi, N., Aleson-Carbonell, L., Pérez-Alvarez, J. A. and Kuri, V. 2005. Antioxidant and antibacterial activities of natural extracts: Application in beef Meatballs. Meat Science. 69: 371-380.

Hayes, J. E., Allen, P., Brunton, N., O’Grady, M. N. and Kerry, J. P. 2010. Phenol composion of in-vitro antioxidant capacity of four commercial phytochemical products: olive leaf extracts (Olea europaea L), lutein, sesame oil and ellagic acid. Food Chemistry. 126: 948-955.

Ikhlas, B., Huda, N. and Noryati, I. 2011. Chemical composition physicochemical properties of meatballs prepared from mechanically deboned quail meat using various types of flour. International Journal of Poultry Science. 10 (1): 30-37.

Jama, N., Muchenje, V., Chimonyo, M., Strydom, P. E., Dzama, K. and Raats, J. G. 2008. Cooking loss components of beef from Nguni, Bonsmara and Angus steers. African Journal of Agriculture Research. 3: 416-420.

Jung, S., Choe, J., Kim, B., Yun, H., Kruk, Z. A. and Jo, C. 2010 Effect of dietary mixture of garlic acid and linoleic acid on ant oxidative potential and quality of breast meat from broilers. Meat Science. 86: 520 -526.

Koushik, P. and Gopal, B. B. 2013. Ocimum sanctum (Tulsi) stem extract mediated size controlled green synthesis of polyshaped gold nanoparticles and its application in catalysis. International Journal of Research in Chemistry and Environment. 3(1): 128-135.

Lee, J. Y. and Kunz, B. 2005. The antioxidant properties of baechukimchi and freeze dried kimchi powder in fermented sausages. Meat Science. 69: 741-747.

Prasad, W., Khamrui, K., Nandal, S. and Badola R 2017. Antioxitative, physico-chemical and sensory attributes of burfi affected by incorporation of different herbs and its comparison with synthetic anti- oxidant (BHA). Journal of Food Science and Technology, 54(12): 3802-3809.

Pandey, G, Madhuri, S. 2010. Pharmacological activities of Ocimum sanctum (Tulsi): a review. International Journal of Pharmaceutical Science Review and Research, 5(1): 6166.

Puri and Singh, H. 2002. Rasayana: Ayurvedic Herbs for Longevity and Rejuvenation. CRC Press, Boca Raton, FL, pp 272280.

Purnomo, A. D. and Rahardiyan, 2008. Indonesian traditional meatball: Review article. Journal of International Food Research. 15: 101-108.

Rubio, B. B., Martinej, M. J., Sinchey, M. D., Garcia-cachan, J., Rovira and Jaime, I. 2007. Study of self-life of a dry fermented sausage Salchichon made from raw material enriched in monounsaturated and polyunsaturated fatty acids and stored under modified atmospheres. Meat Science. 76: 128-137.

Sharma, P., Jha, A. B., Dubey, R. S. and Pessarakli, M. 2012. Reactive oxygen species, oxidative damage and Antioxidative defence mechanism is plants under stressful conditions. Journal of Botany. Volume 2012, Article ID 217037 Vol No.????. 1-26. doi: 10.1155/2012/217037

Sallam, K. I., Ishioroshi, M, and Samejima, K. 2004. Antioxidants and antimicrobial effects of garlic on chicken sausage. Lebensm. Wiss. Technology. 37: 849-855.

Serdaroglu, M., Yldz, T. G. and Abrodimov, K. 2005. Quality of lowfat meatballs containing legume flours as extenders. Meat Science. 70 (1): 99-105.

Singh, V., Amdekar, M. S. and Verma, O. 2010. Ocimum sanctum (tulsi); Pharmacological Activities. International Journal of Recent Scientific Research. WebMD Central Pharmacology. 1(10) WMC0010-46.

Sreelatha, S. and Padma, P. 2009. Antioxidant Activity and Total Phenol Content of Moringa oleifera Leaves in Two Stages of Maturity. Plant Foods and Human Nutrition. 64: 303311.

Verma, A.R., Vijayakumar, M., Mathela, C. S. and Rao C.V. 2009. In vitro and in vivo antioxidant properties of different fractions of Moringa oleifera leaves. Food and Chemical Toxicology. 47: 2196-2201. 